\title{
Enquêtes Ethnobotaniques et Caractérisation des Pratiques Endogènes de Gestion des Ravageurs sur Quelques Sites Maraîchers au Sud-Bénin
}

\author{
Fernand A. Sotondji, \\ Faculté des Sciences et Techniques, Université d'Abomey-Calavi, Bénin \\ Elie Dannon, \\ Enseignant Chercheur en Entomologie Agricole, Université Nationale des \\ Sciences, Technologies, Ingénieries, et de Mathématiques d'Abomey \\ (UNSTIM), Bénin \\ Angelo Cocou Djihinto, \\ Chargé de Recherche (CAMES) \\ à l'Institut National de Recherche Agricole du Bénin \\ Daniel C. Chougourou, \\ Enseignant Chercheur au Département Génie de l'Environnement à l'Ecole \\ Polytechnique d'Abomey-Calavi (EPAC), Bénin
}

\section{Douro Kpindou O. Kobi,}

Chargé de Recherche (CAMES) Chercheur entomologiste à IITA, Bénin

Gildas Zodome,

Faculté des lettres Arts et Sciences Humaines,

Université d'Abomey-Calavi, Bénin

Euloge Adjou,

Enseignant- Chercheur en Technologie

Alimentaire à l'Ecole Polytechnique d'Abomey-Calavi, Bénin

Mohamed M. Soumanou,

Professeur titulaire en Biochimie à l'Ecole

Polytechnique d'Abomey-Calavi, Bénin

Doi:10.19044/esj.2019.v15n30p389 URL:http://dx.doi.org/10.19044/esj.2019.v15n30p389

\section{Résumé}

L'une des contraintes liées à la production maraîchère, est la pression exercée par les insectes ravageurs. Ce travail a consisté en une enquêtes ethnobotaniques et caractérisation des pratiques endogènes de gestion des ravageurs sur quelques sites maraîchers au sud-Bénin, dans le cadre de la formulation des pesticides botaniques pour la gestion des principaux ravageurs de cultures maraîchères. Cette étude est basée sur les caractéristiques socioéconomiques des enquêtés, l'état de connaissances du producteur sur les 
cultures maraîchères, les contraintes et méthodes de lutte des cultures, et les méthodes d'utilisation de la plante. Un questionnaire d'enquête portant sur l'étude ethnobotanique des plantes et la caractérisation des pratiques endogènes de gestion des nuisible a été élaborée et adressé auprès 240 maraîchers sur une période de trois mois (de mai 2018 à Juillet 2018) pour la collecte des données. Ces données ont été soumises à une analyse descriptive et une analyse en composante principale. L'étude a révélé que 12 espèces de légumes sont cultivées par les maraîchers de la zone d'étude à cause de leur cycle assez court et leur préférence par les consommateurs. Ces légumes sont dominés par la grande morelle, le chou, la tomate et la carotte en termes de proportion. Le recours aux pesticides de synthèse non homologués pour le contrôle des ravageurs reste la méthode de lutte la plus utilisée. Les connaissances ethnobotaniques collectées sur quatre plantes à savoir : le neem, le moringa, le papayer et le tabac, au cours de nos enquêtes sont très riches mais détenues par très peu de personnes, majoritairement âgées d'au moins 50 ans $(88,98 \%)$. Ces plantes se répartissent en quatre familles (Caricaceae, Moringaceae, Méliacées, Solanacées) dont les Méliacées sont les plus utilisées. Les parties des plantes les plus utilisées sont les feuilles et les graines. Cette collection de plantes indiquées par les maraîchers dans la lutte contre les ravageurs des cultures maraîchères peut constituer une base des données pour des tests biologiques et des analyses phytochimiques.

Mots clés: Cultures maraîchères, Enquêtes ethnobotaniques, Plantes insecticides, Gestions, Ravageurs 


\title{
Ethnobotanical Surveys and Characterization of Endogenous Pest Management Practices in a Few Market Garden Sites in Southern Benin
}

\author{
Fernand A. Sotondji, \\ Faculté des Sciences et Techniques, Université d'Abomey-Calavi, Bénin \\ Elie Dannon, \\ Enseignant Chercheur en Entomologie Agricole, Université Nationale des \\ Sciences, Technologies, Ingénieries, et de Mathématiques d'Abomey \\ (UNSTIM), Bénin \\ Angelo Cocou Djihinto, \\ Chargé de Recherche (CAMES) \\ à l'Institut National de Recherche Agricole du Bénin \\ Daniel C. Chougourou, \\ Enseignant Chercheur au Département Génie de l'Environnement à l'Ecole \\ Polytechnique d'Abomey-Calavi (EPAC), Bénin
}

\section{Douro Kpindou O. Kobi,}

Chargé de Recherche (CAMES) Chercheur entomologiste à IITA, Bénin

Gildas Zodome,

Faculté des lettres Arts et Sciences Humaines,

Université d'Abomey-Calavi, Bénin

Euloge Adjou,

Enseignant- Chercheur en Technologie

Alimentaire à l'Ecole Polytechnique d'Abomey-Calavi, Bénin

Mohamed M. Soumanou,

Professeur titulaire en Biochimie à l'Ecole

Polytechnique d'Abomey-Calavi, Bénin

\begin{abstract}
One of the constraints related to vegetable production is the insect pests pressure. This work consisted of ethnobotanical surveys and characterization of endogenous pest management practices in a few gardening sites in southern Benin, as part of the formulation of botanical pesticides for the management of major gardening pests. This study is based on the socioeconomic characteristics of the surveyeds, the state of knowledge of the producer on gardening, constraints and methods of the crop control, and methods of the plant use. A survey quiz on the ethnobotanical study of plants
\end{abstract}


and the characterization of endogenous pest management practices was developed and sent to 240 market gardeners over a period of three months (May 2018 to July 2018) for data collection. These data were subjected to a descriptive analysis and a principal component analysis. The study revealed that 12 species of vegetables are grown by market gardeners in the study area because of their rather short cycle and their preference by consumers. These vegetables are dominated by large nightshade, cabbage, tomato and carrot in terms of proportion. The use of unregistered synthetic pesticides for pest control remains the most widely used control method. The ethnobotanical knowledge collected on four plants namely: neem, moringa, papaya and tobacco, during our surveys are very rich but held by very few people, mostly aged at least 50 years $(88,98 \%)$. These plants are divided into four families (Caricaceae, Moringaceae, Meliaceae, Solanaceae) whose Meliaceae are the most used. The most used plant parts are leaves and seeds. This collection of plants indicated by market gardeners in the fight against vegetable crop pests can constitute a database for biological tests and phytochemical analyzes.

Keywords: Market gardening, Ethnobotanical surveys, Insecticidal plants, Management, Pests

\section{Introduction}

Depuis quelques décennies, le maraîchage connaît un essor dans les villes béninoises. En effet, suite aux programmes d'ajustements structurels ayant conduit au gel des recrutements dans la fonction publique dans les années 1987, au pouvoir d'achat assez faible des béninois, au chômage galopant de ces derniers, de nombreux diplômés et sans-emplois se sont lancés dans le secteur du maraîchage qui constitue de nos jours, une véritable activité génératrice de revenu en zones urbaines et périurbaines (Temple et Moustier, 2004; James et al, 2010). La filière cultures maraîchères contribue à assurer la sécurité alimentaire par la production des légumes et assure ainsi la disponibilité en aliments à la population. Elle se développe à côté du secteur tertiaire donnant l'accès au revenu à un grand nombre d'individus et réduisant ainsi le niveau de chômage. Elle contribue à la création de plus de 600000 emplois directs au Bénin (PADAP, 2003). Plusieurs systèmes de production maraîchère ont été répertoriés. Les deux grands systèmes sont les systèmes de production intensive utilisant les pesticides et les engrais chimiques et les systèmes de production biologique. Au Bénin, les premiers sont pratiqués par la grande majorité des producteurs (Akogbéto et al., 2005 ; Ahouangninou et al., 2011).

Dans les villes de Cotonou, de Sèmè-Kpodji et de Grand-Popo, la production maraîchère urbaine ou périurbaine joue un grand rôle dans l'approvisionnement des ménages en fruits et légumes et permet de fournir 
aux populations des villes côtières béninoises, environ $64 \%$ de la consommation annuelle pour certains légumes (Adorgloh-Hessou, 2006 ; Adifon et al, 2015). Ce secteur du maraîchage urbain ou périurbain selon Madjouma et al, (2009), est à considérer comme une solution pour un meilleur approvisionnement en légumes des villes d'Afrique subsaharienne soumises ces dernières décennies à une urbanisation sans précédent.

Cependant, la production maraîchère présente un certain nombre de spécificités par rapport à l'agriculture rurale ou conventionnelle à savoir : nouvelles fonctions marchandes et non marchandes, complexité du droit foncier, fort usage des intrants chimiques, nécessité d'une maîtrise de l'eau, des circuits de commercialisation et d'approvisionnement des produits phytosanitaires de synthèse (Moustier et Mbaye, 1999; Ogouwalé, 2007 ; Adifon et al, 2015). En effet, la volonté de couvrir les besoins alimentaires des villes ou des grandes zones urbaines pousse les maraîchers à recourir à l'utilisation intensive d'intrants chimiques pour le contrôle des ravageurs et la gestion de la fertilité des sols (Doumbia et Kwadjo, 2009 ; Ahouangninou et al., 2011). Or, l'usage intensif des intrants chimiques (produits phytosanitaires de synthèse et engrais minéraux) en production agricole en général et production maraîchère en particulier, n'est pas sans effet sur la qualité sanitaire des cultures maraîchères et sur leur environnement de production. L'utilisation répétitive et massive des intrants chimiques dont notamment les engrais minéraux, les pesticides ainsi que des eaux usées ou de marécages en production agricole sans certaines précautions est préjudiciable aux composants de l'environnement dont le sol, les eaux souterraines, les produits agricoles et la santé humaine (Guédénon, 2009 ; Alapini-Kakpo, 2009 ; Koumolou, 2009). Des méthodes alternatives de lutte présentant moins de risques existent. Il s'agit de l'utilisation des extraits de plantes comme Azadirachta indica, Hyptis suaveolens et Carica papaya et qui sont de fabrication locale. L'introduction de ces biopesticides dans les systèmes de production des légumes est une forme de lutter contre les ravageurs des cultures tout en respectant les principes écologiques, la santé humaine et l'environnement. Elle est considérée comme une partie intégrante d'un système de production durable car les biopesticides permettent de valoriser au mieux les ressources locales, d'améliorer la qualité des produits, de réduire les coûts de production et favoriseraient l'augmentation de la productivité, et par conséquent, l'amélioration des revenus des producteurs (Adétonah, 2007). Mais leur utilisation est très limitée par les producteurs (Adékambi et al., 2010). En effet, même si les biopesticides sont moins dangereux pour les agroécosystèmes et diminuent le risque de présence de résidus dans les aliments produits, certains ont une action moins rapide ou nécessitent un environnement spécifique pour être efficace. Certains pourraient aussi avoir un impact sur des organismes non cibles utilisés dans la lutte biologique (Birch 
et al., 2011). Une substitution des biopesticides aux pesticides chimiques devient une question importante en production maraîchère.

L'objectif de cette étude est d'analyser les contraintes et les méthodes de lutte contre les ravageurs des cultures maraîchères et d'identifier les plantes à effet insecticides utilisées localement par ces derniers, pour des tests biologiques sur certains principaux ravageurs.

\section{1.materiel et Methodes 1.1.Milieu d'étude}

Les sites d'enquêtes ethnobotaniques sont présentés sur la figure (1). Ces sites sont des zones de fortes productions de culture maraîchère. La présente étude a porté sur quatre périmètres marâ̂chers de la zone côtière du Sud-Bénin (Vimas, Houéyiho, Togba et le cordon littoral de Grand-Popo). Le site maraîcher de Vimas est situé dans la commune de Sèmè-Kpodji entre $6^{\circ} 21$ et $6^{\circ} 22$ de latitude Nord et $2^{\circ} 34$ et $2^{\circ} 35$ de longitude Est. Celui de Houéyiho se trouve en plein cœur de la ville de Cotonou entre $6^{\circ} 21$ et $6^{\circ} 22$ de latitude Nord et de $2^{\circ} 23$ à $2^{\circ} 24$ de longitude Est. Il est localisé dans le 12ème arrondissement de la ville de Cotonou et s'étend sur une superficie de 15 ha. Le site maraîcher de Togba est situé dans la commune d'Abomey- Calavi entre $6^{\circ} 19$ et $6^{\circ} 20$ de latitude Nord $2^{\circ} 15$ à $2^{\circ} 18$ longitude. Quant au quatrième site maraîcher, il est situé dans la commune de Grand-Popo au Sud-Ouest Bénin entre $6^{\circ} 12$ à $6^{\circ} 27$ de latitude Nord et $1^{\circ} 37$ à $1^{\circ} 55$ de longitude Est. L'ensemble de la zone d'étude jouit d'un climat du type subéquatorial maritime caractérisé par quatre saisons inégalement réparties dont deux saisons des pluies et deux saisons sèches. Il s'agit d'une grande saison des pluies de mi-mars à mi-juillet, d'une saison sèche de mi-juillet à mi-septembre, d'une petite saison des pluies de mi-septembre à mi-novembre et une grande saison sèche de mi-novembre à mi-mars.

Les températures moyennes mensuelles varient entre $26^{\circ}$ et $27^{\circ} \mathrm{C}$. Les températures maximales journalières les plus élevées dépassent rarement 34 ${ }^{\circ} \mathrm{C}$. Elles sont enregistrées entre février et mars voire avril $\left(32,5^{\circ} \mathrm{C}\right)$, qui se révèlent être les mois les plus chauds de l'année. Les températures les plus basses $\left(24^{\circ} \mathrm{C}\right.$ en moyenne) interviennent en Août. L'humidité reste souvent élevée mais connaît une légère baisse en décembre et en janvier à cause de l'harmattan. La valeur maximale de l'humidité relative mensuelle est de $95 \%$, ce qui constitue un facteur favorable à la corrosion et à la biodégradation. Les moyennes mensuelles varient entre 74,27 et 81,18\%. La moyenne mensuelle des précipitations oscille entre 13,04 mm en janvier et 309,44 mm en juin, avec une moyenne annuelle de 1195,62 mm (Adam et Boko, 1993, Adifon et al, 2015). La morphologie du relief du milieu d'étude est globalement plane mais peut présenter localement des rides et des buttes d'origine éolienne qui s'emboîtent les unes dans les autres. La zone d'étude fait également partie de 
l'ensemble géomorphologique de la zone du littoral du Sud-Bénin qui est un domaine très complexe à cause de la diversité et du dynamisme des éléments qu'ils associent : la mer, les deltas, les lagunes, les lacs, les marécages et les cordons littoraux. Ainsi, le relief du périmètre maraîcher de Houéyiho est morphologiquement hétérogène. Il est basé sur une bande de sable alluvial et d'un ensemble de marécages traversé par deux pistes qui s'acheminent à l'entrée et à la sortie du site. Les différents sites maraîchers étudiés reposent sur un sédiment meuble et s'étend en majorité sur des sols sablonneux acides (sols blancs à tendance podzolique). Ces sols à profil peu évolué sont dominés par des alluvions sableuses et pauvres en éléments chimiques et en matière organique.

\subsection{Choix des sites d'études}

Le choix des périmètres maraîchers objets d'étude se justifie par le fait qu'ils font partie des plus importants sites maraîchers intra-urbains (site de Houéyiho) et périurbains (sites de Sèmè- Kpodji, Togba et de Grand-Popo) au Sud-Bénin. Ils fournissent aux populations de la région côtière, plus de $64 \%$ de la production maraîchère urbaine et péri-urbaine (Adorgloh-Hessou, 2006, Adifon et al., 2015).

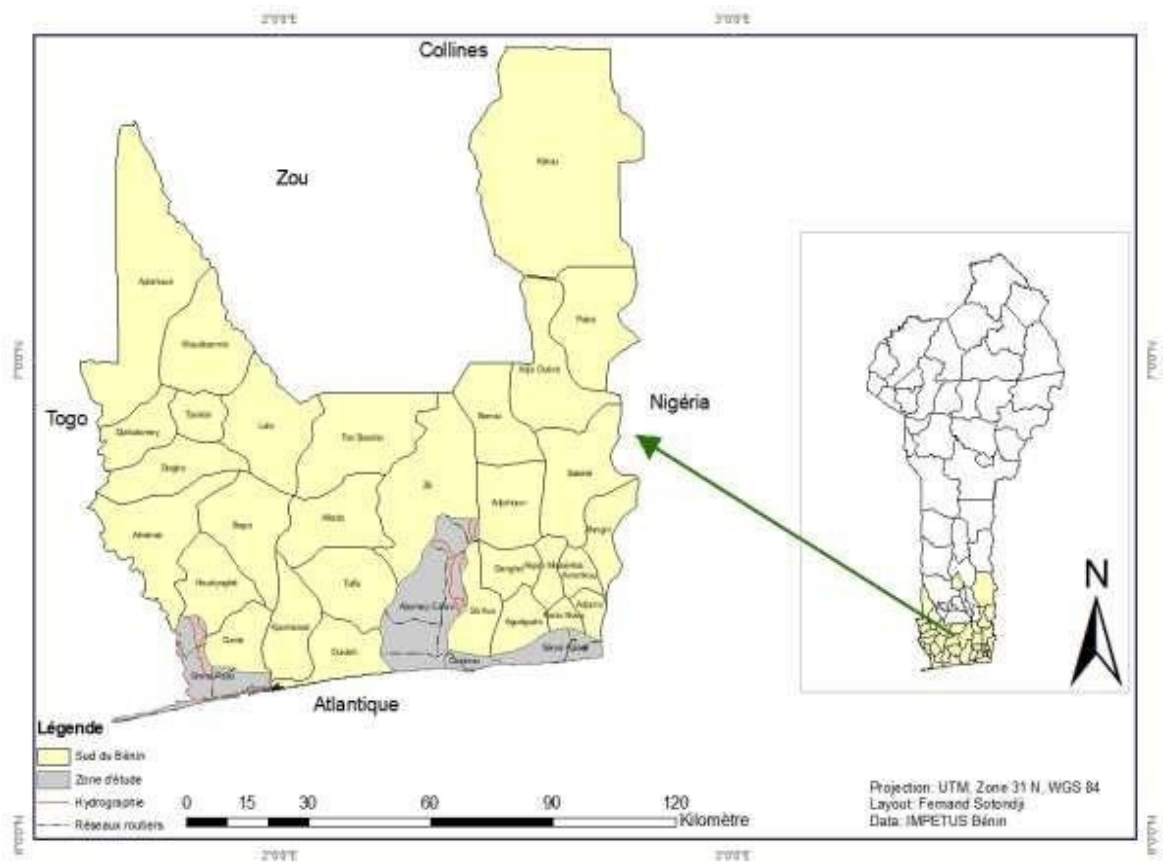

Figure (1): Carte des périmètres maraîchers objets d'étude (enquête SOTONDJI, 2018)

\subsection{Mode d'échantillonnage}

La taille de l'échantillon dans le cadre de cette étude a été calculée en utilisant l'approximation normale de la distribution binomiale proposée par 
Dagnelie en 1998. Ainsi: $\boldsymbol{n}=\mathbf{z} 2 * \boldsymbol{P} *(1-\boldsymbol{P})$ avec $\mathbf{n}:$ Taille de l'échantillon ; $\mathbf{P}$ : Proportion estimative de la population $\boldsymbol{e} 2$ présentant la caractéristique étudiée $(82,2 \%$ ou 0,822$)$. $\boldsymbol{u}$ : Intervalle de confiance $(u=1,96$ pour $95 \%$ de niveau de confiance); $\boldsymbol{e}$ : Marge d'erreur acceptable $(5 \%)$

On a alors : $\boldsymbol{n}=1,96^{2} * 0,822 *(1-0,822)=240$ (taille de l'échantillon)

$$
0,05^{2}
$$

A partir des valeurs de $\mathrm{p}$ issues des résultats de la phase exploratoire de l'étude, 240 producteurs maraîchers au total ont été sélectionnés dans le milieu d'étude. Ils ont été répartis dans les quatre sites maraîchers d'étude en fonction de l'importance des superficies moyennes emblavées en cultures maraîchères et des années d'expérience. Dans chacune des villes et sur chaque périmètre, les maraîchers enquêtés ont été sélectionnés selon un échantillonnage par ciblage.

\subsection{Méthodes et outils de collecte des données}

Les données ont été collectées entre mai et juillet 2018 à l'aide d'un questionnaire semi- structuré qui a permis de recueillir des informations quantitatives et qualitatives.

Une première étape exploratoire a eu lieu sur les quatre sites maraîchers du sud Bénin qui font l'objet d'étude. Elle a eu pour objectif de recenser les plantes à effet insecticide utilisées par ces derniers. La deuxième étape de l'enquête s'est orientée vers les pratiques endogènes de gestions des nuisibles dans les zones d'étude concernées. Les données ont été collectées à l'aide d'un questionnaire individuel.

Les données collectées sont relatives aux caractéristiques sociodémographiques, aux spéculations pratiquées, aux méthodes de lutte endogènes et modernes y compris les méthodes de lutte intégrée, aux produits utilisés pour les traitements phytosanitaires, aux critères de choix des méthodes de lutte, aux contraintes liées à la production maraîchère, à la collecte et à l'utilisation des plantes indigènes à effets insecticides.

En première partie, le questionnaire a permis l'évaluation de la connaissance de quatre plantes à effet insecticide utilisées par les producteurs. La deuxième partie de l'enquête était relative aux méthodes et pratiques utilisées pour la gestion des ravageurs. L'identification des insectes ravageurs a été faite par la présentation de photographies et parfois de spécimens d'insectes. En effet, pour chaque plante hôte et pour chacun des insectes ravageurs, les agriculteurs ont estimé l'importance des dégâts. Pour s'assurer de la connaissance de l'insecte, l'enquêté devait préciser les cultures attaquées par le ravageur et la nature des dégâts qu'il occasionne. 


\subsection{Traitement et analyse des données}

Les données ont été saisies dans le logiciel SPHINX version 5, en suite converties dans le logiciel SPSS version 21 pour le nettoyage et l'épurement. Les analyses descriptives et les tests statistiques ont été réalisés avec le logiciel STATA version 13.

\section{2- Resultats}

\subsection{Caractéristiques socio-démographiques et professionnelles des maraîchers enquêtés}

La production maraîchère se développe à la périphérie des grandes villes africaines. Le Tableau 1 et 1-1 présente les résultats de l'analyse descriptive des variables socio-économiques des maraîchers de la région littorale du Sud-Benin. Les personnes enquêtées sont tous producteurs du maraîchage avec des superficies variées. Les enquêtés ont en moyenne 45 ans d'âge avec 18 ans d'expérience dans le maraîchage, à cause de la fiabilité des données, la majorité des maraichers 70,97\% ont moins de 50 ans. Environ 30 $\%$ d'entre eux n'ont jamais fréquenté l'école, 40,68\% ont atteint le niveau d'éducation primaire et 58,62\% ont atteint le niveau secondaire d'éducation, seuls $0,7 \%$ d'entre eux avaient fait des études supérieures. Ils sont minoritairement analphabètes à moins de $32,2 \%$ contre 67,8 . Les maraîchers sont composés de $28,96 \%$ de femmes contre $74,4 \%$ d'hommes, Ceci prouve l'implication et l'adhésion des femmes dans la production des cultures maraîchères, près de $98,75 \%$ étaient mariés, $1,25 \%$ étaient célibataires et 0,42 \% étaient veufs. La majorité des marâichers $(90,3 \%)$ appartiennent à un groupement/ association. Une grande partie des producteurs $88,47 \%$ avaient la production maraîchère comme activité principale. Environ $(97,1 \%)$ des enquêtés ont répondu aux questionnaires, dont $100 \%$ chez les femmes et 96 $\%$ des hommes, ce pourcentage prouve le dynamisme des enquêteurs à aller à la source des vraies informations.

Tableau 1 : Caractéristiques socio-démographiques des enquêtés

\begin{tabular}{|c|c|c|c|c|c|c|c|}
\hline \multirow[t]{3}{*}{ Variables } & \multirow{3}{*}{ Modalités } & \multicolumn{4}{|c|}{ Pourcentage des répondants ( \%) } & \multirow[b]{2}{*}{$\begin{array}{l}\text { Total } \\
\text { zones } \\
\text { d'étude }\end{array}$} & \multirow[b]{2}{*}{$\begin{array}{l}\text { Test } \\
\text { Khi- } \\
\text { deux }\end{array}$} \\
\hline & & Houéyiho & $\begin{array}{l}\text { Grand } \\
\text { Popo }\end{array}$ & $\begin{array}{l}\text { Sémè } \\
\text { Kpodji }\end{array}$ & Togba & & \\
\hline & & $(n=60)^{1}$ & $(n=60)^{1}$ & $(\mathrm{n}=60)^{1}$ & $(\mathrm{n}=60)^{1}$ & $\begin{array}{l}(\mathrm{N}= \\
240)^{1}\end{array}$ & \\
\hline \multirow{2}{*}{ Sexe } & Homme & 75 & 75 & 43,9 & 92,2 & 74,4 & 6,2 \\
\hline & Femme & 25 & 25 & 56,1 & 9,8 & 28,96 & \\
\hline
\end{tabular}




\begin{tabular}{|c|c|c|c|c|c|c|c|}
\hline Age & $0 \leq$ Age $<50$ & 77 & 66,7 & 70 & 70,2 & 70,97 & 5,23 \\
\hline & $50 \leq$ Age $<70$ & 23 & 33,3 & 28,3 & 29,8 & 28,6 & \\
\hline & Age $\geq 70$ & 0 & 0 & 1,7 & 0 & 0,42 & \\
\hline \multirow{5}{*}{$\begin{array}{l}\text { Niveau } \\
\text { d'instructio } \\
\text { n }\end{array}$} & Primaire & 29,2 & 20,6 & $62,8^{2}$ & 50 & 40,65 & $8.1^{* *}$ \\
\hline & 1er cycle & 35,3 & 54,2 & 37,2 & 45,2 & 42,97 & \\
\hline & 2nd cycle & 41,2 & 16,7 & 0 & 4,8 & 15,65 & \\
\hline & Universitaire & 2,9 & 0 & 0 & 0 & 0,7 & \\
\hline & \begin{tabular}{|l|} 
Formati \\
on \\
technin
\end{tabular} & 24,5 & 13,8 & 10,1 & 20,2 & 17,15 & \\
\hline \multirow[b]{2}{*}{ Alphabétisation } & Alphabétisés & 55,7 & 74,6 & 75 & 64,4 & 67,8 & 2,81 \\
\hline & \begin{tabular}{|l|} 
Non \\
alphabétis \\
és \\
\end{tabular} & 44,3 & 25,4 & 25 & 34,6 & 32,2 & \\
\hline \multirow{3}{*}{$\begin{array}{l}\text { Situation } \\
\text { matrimoniale }\end{array}$} & Marié & 96,7 & 100 & 98,3 & 100 & 98,75 & \\
\hline & Célibataire & 3,3 & 0 & 1,7 & 0 & 1,25 & \\
\hline & Veuve (f) & 0 & 0 & 1,7 & 0 & 0,42 & \\
\hline \multirow{3}{*}{$\begin{array}{l}\text { Taille du } \\
\text { ménage } \\
\text { du } \\
\text { maraîche } \\
r\end{array}$} & $\begin{array}{c}1 \leq \text { nombre } \\
\text { personnes }<5\end{array}$ & 56,7 & 65,4 & 45,2 & 54,8 & 55,52 & 2,72 \\
\hline & $\begin{array}{l}5 \leq \text { nombre de } \\
\text { personnes } \\
<10\end{array}$ & 39,5 & 28 & 41,4 & 38,9 & 36,95 & \\
\hline & $\begin{array}{lr}\text { Nombre } & \text { de } \\
\text { personnes } & \geq \\
10 & \\
\end{array}$ & 3,8 & 6,6 & 13,4 & 6,3 & 7,75 & \\
\hline
\end{tabular}

Tableau 1-1 : Caractéristiques professionnelles des enquêtés

\begin{tabular}{|l|l|l|l|l|l|l|l|}
\hline $\begin{array}{l}\text { Expérien } \\
\text { ce } \\
\text { (années) }\end{array}$ & $\begin{array}{l}\text { Entre 10 et } \\
20 \text { ans }\end{array}$ & 71,4 & 67,7 & 95,3 & 74,4 & 77,2 & 12,39 \\
\cline { 2 - 7 } & $\geq 20$ ans & 28,6 & 32,3 & 4,7 & 26,6 & 23,05 & \\
\hline
\end{tabular}




\begin{tabular}{|l|l|l|l|l|l|l|l|}
\hline \multirow{2}{*}{$\begin{array}{l}\text { Appartenance } \\
\text { à une } \\
\text { organisation } \\
\text { paysanne }\end{array}$} & Appartenance & 71,1 & 98,3 & 96,7 & 94,7 & 90,2 & 7,97 \\
\cline { 2 - 7 } & $\begin{array}{l}\text { Non } \\
\text { appartenan }\end{array}$ & 27,9 & 1,7 & 3,3 & 5,3 & 9,6 & \\
\hline $\begin{array}{l}\text { Activité } \\
\text { principa } \\
\text { le }\end{array}$ & OUI & 87,9 & 85,2 & 89,6 & 91,2 & 88,47 & 31,21 \\
\hline
\end{tabular}

2.2 Spéculations végétales produites et revenus de l'exploitation sur les sites

Plusieurs spéculations végétales ont été recensées au cours de nos enquêtes, sur les sites de Houéyiho, Grand Popo, Sèmè-kpodji et de Togba (Figure 2 et Tableau 2). Il s'agit aussi bien d'espèces locales qu'exotiques. Les cultures les plus fréquentes ont été la grande morelle (Solanum macrocarpon), le chou (Brassica oleracea), la tomate (Lycopersicon esculentum, l'amarante (Amaranthus hybridus), la vernonia (Vernonia amygdalina), la laitue (Lactuca sativa), et la carotte (Daucus carota),

Les producteurs en général vendent leur production aux revendeuses détaillantes qui viennent s'approvisionner directement sur les exploitations. En ce qui concerne les revenus tirés de la production, la moyenne annuelle était de (941855 F CFA) 2018 dont 85,22\% de ces revenus provenaient du maraîchage chez les femmes et 79,47 chez les hommes sans la prise en compte de la partie autoconsommée et des pertes dans la production estimées à $86 \%$, $77 \%, 63 \%$, respectivement sur la grande morelle, le chou, et de la tomate, si aucun traitement n'est fait, ces pertes sont dues en grande partie à des ravageurs ( les insectes, les nématodes, les champignons, etc .). Plus de 9 enquêtés sur 10 ont fait les principales cultures maraîchères mais à des fins purement commerciales. Les principales spéculations mises en place sur les différents périmètres maraîchers étudiés dans les villes de, Cotonou (Houéyiho), Grand-Popo (Agoué), et Sèmè- Kpodji ( Site de Vimax) et d'Abomey-Calavi (Togba) sont présentés dans la figure 2. L'analyse de la figure 2 révèle que sur les 12 espèces de cultures légumières recensées, la grande morelle, le chou et la tomate sont les plus produits par la population enquêtée en terme de proportion. Il ressort du tableau 2 après l'analyse des résultats que le chou, la tomate, le piment et la carotte sont plus cultivés à Grand Popo que sur les autres sites qui ont fait l'objet d'étude. 


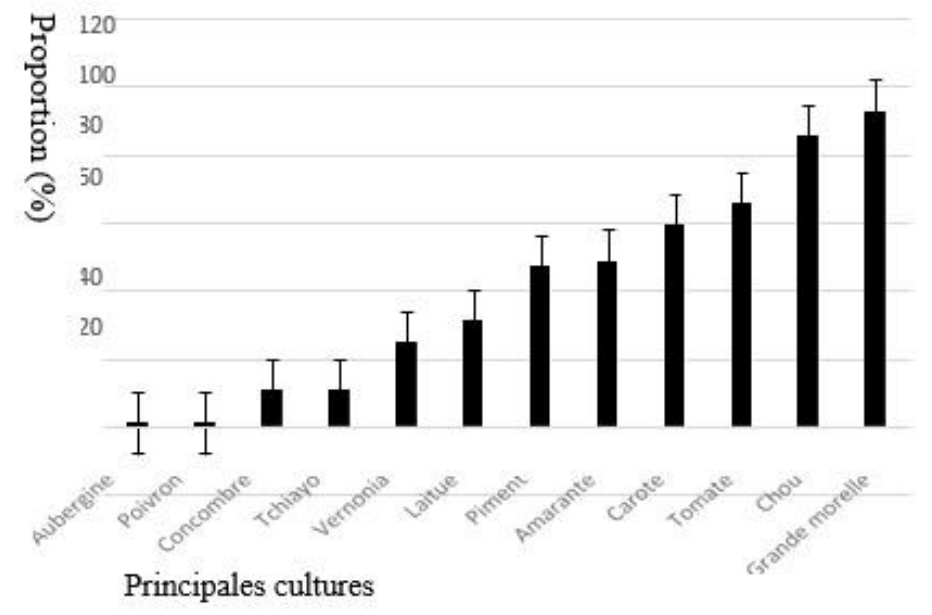

Figure 2: Principales cultures maraîchères sur les sites

Variétés

\begin{tabular}{ccccc} 
Houéyiho & Grand Popo & $\begin{array}{c}\text { Sémè } \\
\text { Kpodji }\end{array}$ & Togba & $\begin{array}{c}\text { Total zone } \\
\text { d'étude }\end{array}$ \\
\hline$(\mathrm{n}=60)$ & $(\mathrm{n}=60)$ & $(\mathrm{n}=60)$ & $(\mathrm{n}=60)$ & $(\mathrm{N}=240)$ \\
\hline 6,2 & 15 & 3 & 15,6 & 9,95 \\
0,6 & 0,7 & 0,7 & 0,4 & 0,6
\end{tabular}

Autres à p

0

0

0

0

0

$\begin{array}{cccccc}\text { Carote } & 11,3 & 10,7 & 15,3 & 13,1 & 12,6 \\ \text { Chou } & 10 & 20 & 20 & 19,9 & 17,47 \\ \text { Concombre } & 8 & 0,7 & 4 & 1,4 & 3,5 \\ \text { Grande mor } & 16,4 & 20,3 & 16 & 20,2 & 18,22 \\ \text { Laitue } & 12,4 & 4,7 & 2 & 6,7 & 6,45 \\ \text { Piment } & 5,8 & 9 & 18 & 5 & 9,45 \\ \text { Poivron } & 0,7 & 0,3 & 0,7 & 0,3 & 0,51 \\ \text { Tchiayo } & 7,8 & 1,3 & 0,4 & 0,4 & 2,45 \\ \text { Tomate } & 6,9 & 13,3 & 18 & 13,5 & 13,02 \\ \text { Vernonia } & 13,9 & 4 & 2 & 3,5 & 5,85 \\ \text { Test Khi-deux } & 36,5 & & & & \end{array}$




\subsection{Les ravageurs et leurs dégâts}

Dans toutes les zones d'étude, les maraîchers ont une bonne connaissance des ravageurs et des dommages qu'ils causent aux cultures.

Les pratiques paysannes de lutte contre les ravageurs de culture sont déterminées par une série de paramètres visant à permettre aux producteurs d'atteindre les objectifs liés à la sécurité alimentaire de son ménage et la maximisation du profit. Ainsi, pour comprendre les raisons qui justifient la prise de décision chez le producteur, il est nécessaire de combiner les différents facteurs susceptibles d'influencer positivement ou négativement sa prise de décision. De plus, la connaissance du poids de chacun des facteurs qui influencent la prise de décision permettra d'identifier les indicateurs clés sur lesquels devra se baser la recherche pour l'amélioration qualitative et quantitative de la production maraîchère. Les dégâts observés sans aucun traitement phytosanitaire signaler par les producteurs qui ont porté leurs choix sur les principales spéculations au cours de nos enquêtes, ainsi que les principaux ravageurs rencontrés sur les cultures maraîchères et leurs dégâts sont respectivement représentés dans la figure 3 et Tableau 3.

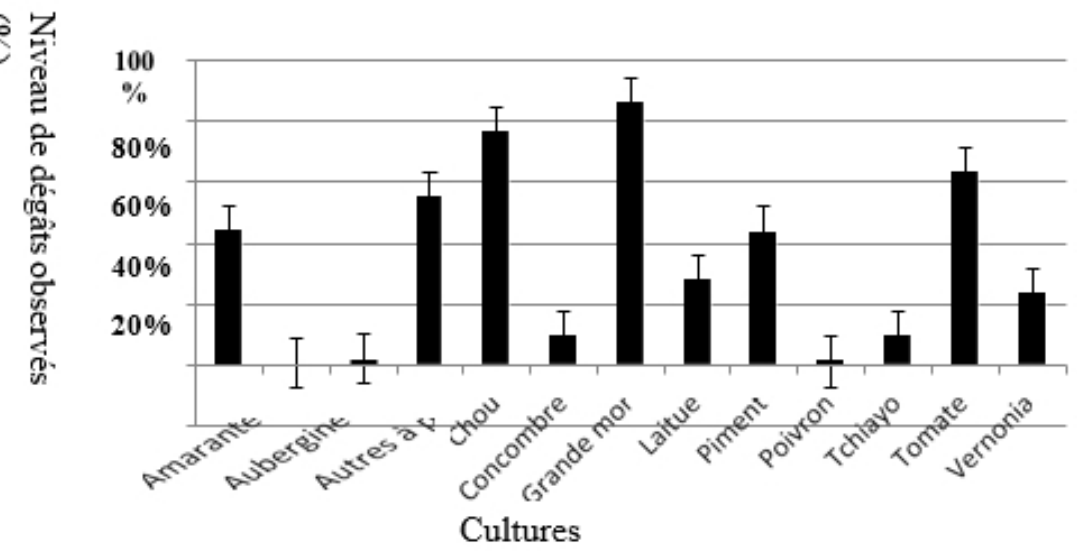

Figure 3: Dégâts causés par les ravageurs sur les cultures emblavées.

Tableau 3: Les principaux ravageurs rencontrés sur les cultures maraîchères et leurs dégâts

\begin{tabular}{|l|l|l|}
\hline Ravageurs & Principales cultures attaquéés & Dégâts \\
\hline Chenilles & $\begin{array}{l}\text { Chou, tomate, grande morelle, laitue, } \\
\text { oignon, concombre, poivron, piment }\end{array}$ & $\begin{array}{l}\text { Perforation des feuilles et } \\
\text { tiges }\end{array}$ \\
\hline Pucerons & Chou, grande morelle, tomate, laitue, carotte & $\begin{array}{l}\text { Taches sur les feuilles } \\
\text { chlorose, nécrose }\end{array}$ \\
\hline $\begin{array}{l}\text { Larves et adultes de } \\
\text { certains coléoptères }\end{array}$ & Tchao, grande morelle, concombre & $\begin{array}{l}\text { Perforation des feuilles et de } \\
\text { certaines racines }\end{array}$ \\
\hline Criquets & Toutes les cultures & Coupures des feuilles et des tiges \\
\hline
\end{tabular}




\begin{tabular}{|l|l|l|}
\hline Nématodes & Tomate, grande morelle, laitue, piment & $\begin{array}{l}\text { Galles, nodules sur les } \\
\text { racines }\end{array}$ \\
\hline Champignons & Tomate, oignon, piment, carotte & $\begin{array}{l}\text { Pourriture, flétrissement et } \\
\text { jaunissement des feuilles }\end{array}$ \\
\hline
\end{tabular}

\subsection{Les méthodes de lutte}

L'utilisation des produits phytosanitaires reste et demeure la principale méthode de lutte contre les ravageurs des légumes. En effet, près de $98 \%$ des maraîchers sur l'ensemble des sites utilisent des produits phytosanitaires pour contrôler les ravageurs. Les traitements phytosanitaires s'effectuent durant tout le cycle des cultures c'est-à-dire depuis le repiquage, la croissance végétative des plants de légumes jusqu'à leurs récoltes. Les fréquences de traitements des cultures sont fonction de la sensibilité de chaque espèce de légume aux attaques parasitaires et de la saison. La fréquence d'utilisation des pesticides varie également d'un producteur à un autre. Il a été observé que pour $80 \%$ des enquêtés ayant des connaissances sur l'importance du respect des normes en matière de qualité sanitaire de légumes, les traitements phytosanitaires interviennent lorsque les attaques parasitaires des légumes se font sentir. Par contre, pour les 20 \% des maraîchers, en l'occurrence ceux ayant une superficie agricole élevée, la fréquence des traitements des légumes est liée au souci d'une bonne productivité et non au seuil de pression parasitaire nécessitant un traitement. Ces derniers effectuent des traitements plutôt préventifs.

\subsection{Contraintes et Solutions}

En dehors des objectifs visés par nos enquêtes sur le terrain ; les maraîchers rencontrent diverses difficultés au niveau des plants. Quelques approches de solutions ont été énumérées par ces derniers.

\section{Au niveau des racines du plant des cultures}

Plus de 93\% des producteurs ont constaté le desséchement de jeunes plants dans leurs champs. Ce phénomène est plus constaté sur le site de Houéyiho, dont 99,2\%, des producteurs en sont victimes surtout sur la culture de la grande morelle et de la tomate.

La lutte mécanique et la lutte chimique sont les mesures utilisées par les producteurs pour faire face à ce problème. La lutte mécanique (extirpé) est utilisée par les producteurs sur tous les sites enquêtés. Quant à la lutte chimique, elle est utilisée à hauteur $30 \%$ par la population enquêtée.

\section{Au niveau des tiges du plant}

Des attaques sévères des tiges par des insectes ont été observées notamment par la destruction des parties tendres (62\%), la section des ramifications $(57 \%)$ et la destruction des apex (89\%). Les producteurs ont observé de dommage sur la tige, et se plaignent des plants de cultures tombés. 
$35 \%$ ont constaté la perforation des tiges ; $27 \%$ observent la destruction des feuilles et la section des ramifications est observée par $57 \%$ mais intense sur certains sites.

La réponse aux attaques fait appel à des mesures de lutte telle la lutte physique, la lutte chimique (LAMBDA SUPER 25 EC, etc...) à hauteur de $37,3 \%$ et la lutte biologique (extrait aqueux de neem) est utilisée par 4,6\%. La lutte physique est plus développée par la population enquêtée environ 98,7\%.

\section{Au niveau des inflorescences des plants}

La majorité des producteurs (84\%) a constaté la chute des inflorescences au niveau des plants, $86 \%$ pour la destruction des inflorescences. 59\% des producteurs affirment faire face à la pourriture des inflorescences. La chute des inflorescences est plus constatée sur la grande morelle $(81 \%)$, la tomate $(74 \%)$, le chou $(67 \%)$, et l'amarante $(55 \%)$. Face à ces dégâts trois mesures sont principalement utilisées par les producteurs. Il s'agit entre autres de la lutte physique (extirpé); la lutte chimique (PACHA , CALFOS 500 EC...) et de lutte biologique (extrait aqueux de neem). La lutte chimique est la plus utilisée sur tous les sites sillonnés $(98,2 \%)$; la lutte physique est inexistante sur les sites de Houéyiho et de Grand Popo. La lutte chimique est plus pratiquée Houéyiho $(99,4 \%)$ comparativement aux autres communes. Les producteurs identifient les dégâts par la présence d'insectes et la perforation des feuilles ou des boutons floraux. La majorité des producteurs (77\%) estime que ces mesures de luttes ne sont pas efficaces et polluent l'environnement.

\section{Au niveau des fleurs}

La perforation des fleurs a été observée chez $96 \%$ des producteurs interviewés dont les plus élevés s'observent sur la culture de la grande morelle $(97,4 \%)$, l'amarante $(88,5 \%)$, le chou $(83,7 \%)$, la tomate $(78,5 \%)$. Seulement $56 \%$ des producteurs ont observé des tâches sur les fleurs. En outre, $95 \%$ ont constatés la destruction des fleurs dont la grande morelle $(91,5 \%)$; le chou $(89,7 \%)$; l'amarante $(81,6 \%)$ et la tomate $(78,6 \%)$ sont en tête en termes de pourcentage. $77 \%$ ont constaté la présence des excréments sur les fleurs. $98 \%$ des producteurs ont observé la présence des insectes sur les fleurs et $74 \%$ de l'avortement au niveau des fleurs.

Pour faire face à ses dégâts, certaines mesures de luttes sont utilisées par les producteurs. Il s'agit entre autres de la lutte physique (extirpé); lutte chimique (lambda super $25^{\circ} \mathrm{C}$ EC, K-OPTIMAL...) et de lutte biologique (extrait aqueux de neem). La lutte physique est presque inexistante sur tous les sites à ce stade (2,1\%). La lutte chimique est plus pratiquée à $(98,8 \%)$.

\subsection{Quelques produits phytosanitaires recensés sur les sites}

Les produits phytosanitaires utilisés par les maraîchers de la zone d'étude sont de diverses formulations. Les concentrés émulsionnables ou, les 
poudres mouillables et les suspensions concentrées sont les plus utilisés par les enquêtés. Au terme du recensement des produits phytosanitaires, des emballages et des caractéristiques de ces derniers, les pesticides utilisés par les maraîchers appartiennent à six sous familles chimiques à savoir les Spinozoïdes; les Pyrétrinoïdes ; les Néonicotinoïdes, les Carbonates, les Benzimidazoles et les Benzènedicarboxamides (Tableau 4).

Tableau 4: Liste et caractéristiques des produits phytosanitaires recensés sur les sites maraîchers de la zone d'étude

\begin{tabular}{|c|c|c|c|c|}
\hline $\mathbf{N}^{\circ}$ & Nom commercial & Matières Actives & Nature & Classes chimiques \\
\hline 1 & $\begin{array}{c}\text { LAMBDA SUPER } \\
25 E C\end{array}$ & $\begin{array}{c}\text { Lambda Cyhalothrine } \\
25 / 1\end{array}$ & Insecticides & Pyréthrinoide \\
\hline 2 & LASER 480 & Spinosad $480 \mathrm{~g} / \mathrm{l}$ & Insecticide & Spinozoide \\
\hline 3 & PACHA & Acétamiprid $10 \mathrm{~g} / \mathrm{l}$ & Insecticide & Néonicotinoïde \\
\hline 4 & CALFOS $500 \mathrm{EC}$ & Profenofos 500g/ & Insecticide & Acaricides \\
\hline 5 & CALTHIO I 350 & $\begin{array}{c}\text { Imidacloprid 250g/l } \\
+ \\
\text { Thiram } 100 \mathrm{~g} / \mathrm{l}\end{array}$ & Insecticide & Fongicides \\
\hline 6 & K-OPTIMAL & Acétamiprid 20g/l & Insecticide & \\
\hline 7 & CYPER-D & Cyperméthrine $10 \mathrm{~g} / \mathrm{l}$ & Insecticides & Pyréthrinoïde \\
\hline 8 & MANCOZEB & $750 \mathrm{~g}$ pour 1001 & Insecticides & Fongicides \\
\hline 9 & BIOBITE & Bacillus thuringiensis & Insecticides & \\
\hline 10 & DEFCAL 8 EC & Pyraflufen ethyl $8 \mathrm{~g} / 1$ & Herbicide & \\
\hline 11 & DIAFURAN & Carbofuran & Nématicide & Carbamate \\
\hline 12 & TOPSIN M & $\begin{array}{c}\text { Méthylthiophanate } \\
700 \mathrm{~g} / 1\end{array}$ & Fongicide & Benzimidazole \\
\hline 13 & CYPERCAL 50 EC & Cyperméthrine $50 \mathrm{~g} / 1$ & Insecticides & Pyréthrinoïde \\
\hline 14 & CYDIM & Cyperméthrine $136 \mathrm{~g} / \mathrm{l}$ & Insecticides & Pyréthrinoïde \\
\hline 15 & DECIS & Deltaméthrine $13 \mathrm{~g} / \mathrm{l}$ & Insecticides & Pyréthrinoïde \\
\hline 16 & THIAN 1750-TEQ & Flubendiamide $100 \mathrm{~g} / \mathrm{l}$ & Insecticides & $\begin{array}{l}\text { Benzène- } \\
\text { dicarboxamide }\end{array}$ \\
\hline 17 & LION-FORCE & $\begin{array}{c}\text { Cypermethrin 30g/l } \\
+ \\
\text { Dimethoate } \\
250 \mathrm{~g} / \mathrm{l}\end{array}$ & Insecticides & Nématicides \\
\hline 18 & CALLOMIL PLUS & $50 \mathrm{~g} / 1$ & Fongicide & \\
\hline 19 & KINIKINI & Cyfluthrine $9,6 \mathrm{~g} / \mathrm{l}$ & Insecticides & Pyréthrinoïde \\
\hline 20 & LAMBDACAL P 315 EC & $\begin{array}{l}\text { Lambda cyhalothrine } \\
15 \mathrm{~g} / 1+\text { Profénofo }\end{array}$ & Acaricides & \\
\hline
\end{tabular}

Source: Enquête sur terrain 2018 


\subsection{Plantes insecticides utilisées dans la protection des cultures maraichères sur les sites enquêtés}

La figure 4 montre que dans la zone d'étude, les paysans ont souvent recours aux plantes locales pour la protection de leurs cultures maraîchères les enquêtes ethnobotaniques effectuées ont révélé l'usage de 4 familles de plantes à effet insecticide (Caricaceae, Moringaceae, Méliacées, Solanacées,) dont les Méliacées sont les plus utilisées. Le neem est plus utilisé sur le site maraîcher de Togba dans la commune d'Abomey-Calavi que sur les autres sites maraîchers enquêtés. Par ailleurs le moringa et le papayer sont utilisés respectivement à proportion égal sur le site de Houèyiho (Cotonou) et de Togba (Abomey-Calavi). Le tabac et le papayer sont plus utilisés dans la commune de Sèmè Kpodji que sur les autres sites (figure 4). Les parties des plantes les plus utilisées sont les feuilles et les graines (Figures 5), ces plantes sont utilisées sous forme d'extrait aqueux. Près de $78 \%$ des maraîchers enquêtés sur le site de Houèyiho, qui utilisaient les pesticides botaniques faisaient des extraits aqueux des feuilles, Les feuilles sont les plus utilisées, ensuite les feuilles associées avec les graines sont moyennement utilisées sur les sites enquêtés. Cette collection de plantes indiquées dans la lutte contre les ravageurs de culture maraîchères peut constituer une base des données pour des études sur les tests biologiques et les analyses phytochimiques.

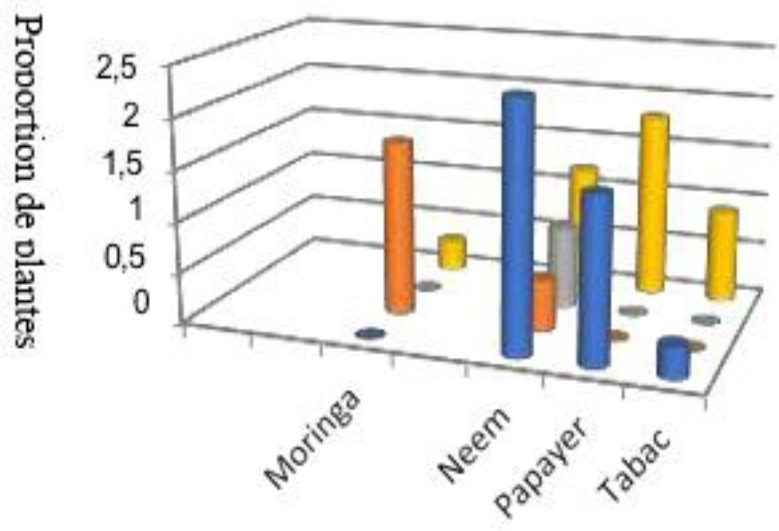

Abomey-Calavi Col \%

Cotonou Col \%

Grand-popo Col \%

Seme-kpodji Col \%

Nom de plantes utilisées

Figure 4: Quelques plantes insecticides utilisées dans la protection des cultures maraîchères au Bénin 


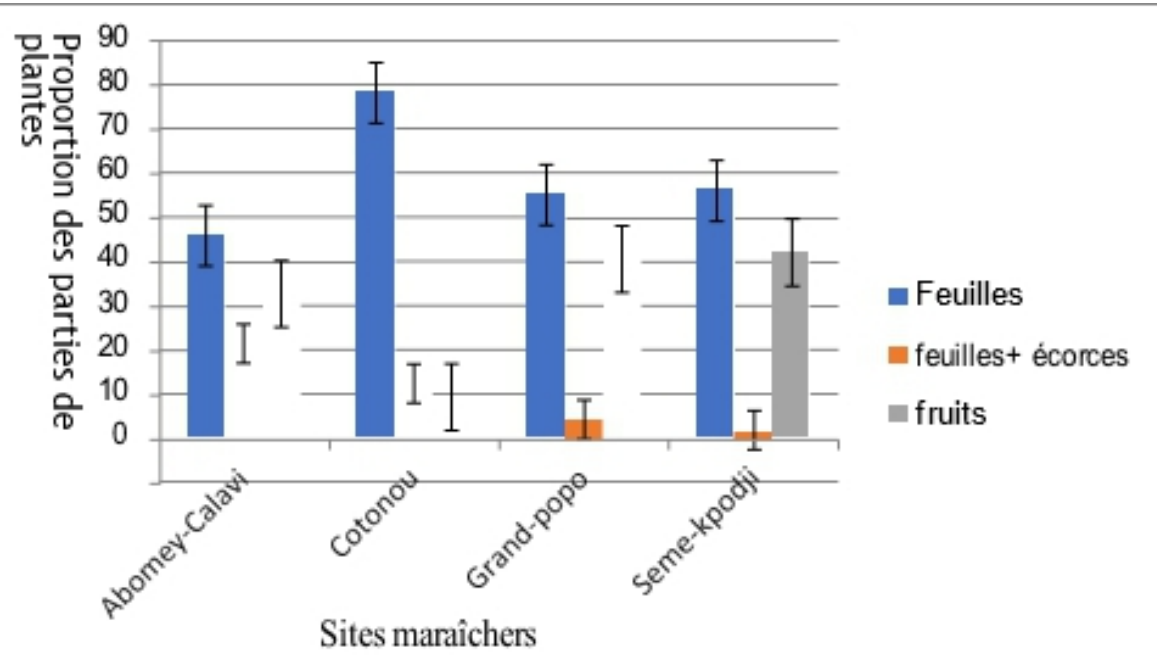

Figure 5: Différentes parties des plantes à effet insecticide utilisées sur les sites prospectés au Bénin

\section{Discussion}

Les maraîchers pour la plupart n'ont pas un niveau élevé et exploitent des petites superficies pour leur activité de production. La grande majorité des producteurs avaient la production maraîchère comme activité principale. Les résultats de travaux de Broutin et al. (2005) au Sénégal sont proches de ceux obtenus au cours de cette étude. Ces auteurs ont rapporté que l'activité maraîchère constitue la principale source de revenu pour $85,23 \%$ de maraîchers et la seule source de revenu pour $34,09 \%$ d'entre eux. L'expérience moyenne en maraîchage était de 15 à 28 ans. Ceci provient du fait que beaucoup de jeunes gens ayant acquis des expériences dans le maraîchage auprès de leurs parents sont restés dans cette activité à cause de la précarité de l'emploi ou des échecs scolaires. Les maraîchers livrent pour la plupart de leur production aux vendeuses détaillant qui les vendent directement aux consommateurs. Très peu de grossistes ou négociants interviennent dans le circuit. Broutin et al. (2005) ont rapporté que les circuits de commercialisation dans le maraîchage au Sénégal sont plus souvent courts (vente directe ou un seul intermédiaire). Selon Moustier (1997). L'activité permet aux maraîchers d'engranger des revenus suffisants pour subvenir aux besoins de leurs foyers. La moyenne annuelle des revenus tirés de la production, était de (941855) F CFA/an par producteur en 2018, soit 78487,9 F CFA par mois. Le maraîchage permet aussi aux femmes des villes de faire la commercialisation des légumes frais, de les mettre à la disposition des consommateurs et de faire des profits pour ainsi subvenir aux besoins de leurs enfants. Selon Tokannou et Quenum (2007), les producteurs qui s'adonnent au maraîchage appartiennent à la catégorie sociale pauvre. Les revenus 
annuels tirés de la production (941855) F CFA/an sont comparables à ceux rapportés par Lemay-Boucher et Dagnelie (2012) chez des habitants de trois quartiers pauvres de Cotonou en 2004 qui étaient en moyenne de 85360 F CFA par mois.

Toute une gamme d'espèces de légumes sont cultivées : légumes feuilles (amarante, grande morelle, chou, laitue etc), fruits (tomate, piment, poivron, concombre, etc), à racines (carotte, betterave, radis) à bulbes (oignon) dans l'ensemble des sites d'étude. Cette gamme variée et multiforme de légumes recensés en maraîchage dans la région littorale au Sud-Bénin est conforme aux travaux de Cissé et al., (2001) ; Diao (2004) et Madjouma et al, (2009). En effet, ces auteurs ont étudié respectivement les systèmes de culture des maraîchers de la région dakaroise au Sénégal et de la ville de Lomé au Togo et ont montré que les maraîchers de ces villes cultivent plusieurs dizaines d'espèces de légumes. L'étude révèle en outre que la production de légumes sur les différents sites est permanente et intensive. L'option d'une production permanente et intensive de légumes adoptée par les maraîchers s'explique d'une part par la forte demande en produits alimentaires frais des ménages urbains et d'autre part en raison de la rentabilité de cette activité qui constitue la principale source de revenus pour la plupart des maraîchers enquêtés.

La contrainte majeure à cette activité est l'attaque des ravageurs de cultures et des maladies dues aux champignons, les autres contraintes étant les problèmes d'irrigation, de fertilité des sols et d'écoulement des produits, la disponibilité de terres, les mauvaises herbes et l'accès aux intrants. Les maraîchers pour la plupart, ne conçoivent pas le fait de produire des légumes et de faire des profits sans pesticides chimiques. La production est caractérisée par l'utilisation massive de pesticides chimiques dans le contrôle des bioagresseurs. L'usage des pesticides pour lutter contre les ravageurs ou pour atténuer la pression parasitaire est également très répandu dans la zone d'étude. Des constats similaires ont été rapportés par Cissé et al, (2001) au Sénégal, Doumbia et Kwadjo (2009) dans la ville d'Abidjan et dans ses banlieues, Kanda et al, (2009 et 2013) au Togo, ainsi que par Ahoungninou et al, 2011, dans la Commune de Tori au Sud-Bénin. Ces résultats confirment ceux de (James et al., 2005), que les maraîchers béninois utilisent pour la protection du chou, 46 litres de pesticides chimiques concentrés en 19 applications par hectare, une dépendance du maraîchage périurbain aux pesticides chimiques a été également rapportée au Bénin par (Akogbéto et al., 2005). L’utilisation de ces pesticides chimiques a des conséquences sur la santé humaine (Ton et al., 2000). Les conséquences environnementales de l'utilisation des pesticides au Bénin ont été documentées dans les travaux de Pazou et al. (2006a) et Pazou et al. (2006b). Les biopesticides disponibles sur le marché LASER 480 SC (Spinosad $480 \mathrm{~g} / \mathrm{L}$ ) et le BIOBIT (Bacillus thuringiensis) n'arrivent toujours pas à éliminer tous les ravageurs détruisant les cultures marâ̂chères. Les 
biopesticides sont moins dangereux pour les agroécosystèmes et diminuent le risque de présence de résidus dans les aliments produits, mais certains ont une action moins rapide ou nécessitent un environnement spécifique pour être efficaces ou pourraient avoir un impact sur des organismes non cibles utilisés dans le biocontrol (Birch et al., 2011). Pour les maraîchers, leur production ne serait pas compétitive sur le marché sans usage des pesticides chimiques. La production est surtout destinée au marché local sur lequel la plupart des consommateurs n'accordent pas grande importance à la qualité chimique des légumes produits. Ces consommateurs ne seraient pas selon eux, disposés à payer plus pour avoir des légumes produits biologiquement. Ceci est aussi constaté dans d'autres villes de l'Afrique de l'ouest. Les consommateurs à Thiès au Sénégal n'utilisent jamais les critères portant sur les modes de production des légumes dans les critères de perception de la qualité rapportent Broutin et al. (2005). Mais les travaux de Coulibaly et al., (2011) rapportent que les consommateurs de choux et de tomates au Bénin et au Ghana sont disposés à payer une prime de plus de $50 \%$ pour avoir des légumes produits sans pesticides chimiques. Selon ces travaux, les critères de sélection des consommateurs de légumes sont l'innocuité du produit, sa couleur, son poids et sa fraîcheur.

L'utilisation systématique des pesticides de synthèse par les maraîchers de la zone d'étude malgré les sensibilisations initiées à leur intention dans le cadre de divers programmes ou projets pour des méthodes alternatives et plus respectueuses de l'environnement se justifie selon eux par le fait que cette méthode est plus efficace et permet d'obtenir une meilleure productivité des légumes. En effet, selon Assogba et al, 2007 et Adifon et al, 2015 , le recours systématique aux pesticides permet aux petits producteurs de garantir une bonne production face à des conditions écologiques favorables aux déprédations. Ce qui constitue des risques de pollution pour l'environnement. Par ailleurs, très peu des maraîchers utilisent des pesticides botaniques comme les feuilles de neem, de papayer, moringa et des feuilles de tabac sur les différents sites d'étude. De façon générale les plantes appartenant à des familles d'espèces reconnues pour leur action pesticide sont largement utilisées par les paysans. Le Neem (Azadirachta indica) appartient à la famille des Méliacées qui comprend 14 genres (Pennington et al., 1981) est le plus largement utilisé. Cela confirme les résultats des travaux de Gnago et al. (2010), en traitant les parcelles de cultures (chou et gombo) avec l'extrait de graines d'A. indica ont remarqué que les populations de chenilles et de pucerons étaient moins importantes sur les parcelles traitées que sur celles non traitées. Des travaux similaires ont révélé qu'un extrait de feuilles d'A. indica a une forte activité insecticide (mortalité > $95 \%$ ) et réduit considérablement la fécondité de M. persicae (Mondédji et al., 2014a). Ces plantes supérieures couramment utilisées en culture maraîchère au Bénin méritent d'être étudiées 
en vue de mettre en évidence les propriétés biologiques des tanins qu'elles renferment.

\section{Conclusion}

Cette étude a permis de donner un aperçu général des cultures maraîchères par des enquêtes socio-économiques et ethnobotaniques sur les plantes à effet insecticide auprès des maraichers. La production de légumes sur le site intra-urbain de Houéyiho dans la ville de Cotonou et sur les sites maraîchers situés sur le cordon littoral dans les Communes de Sèmè-Kpodji, de Togba et de Grand-Popo au Sud-Bénin contribue sensiblement à l'approvisionnement des ménages urbains en légumes. La production de légumes constitue par ailleurs, la principale source de revenu des maraîchers des sites d'étude et la filière fait intervenir différentes catégories d'acteurs (maraîchers, commerçantes, distributeurs d'intrants agricoles, importateurs des produits phytosanitaires, etc.) qui tirent également une partie ou la totalité de leur revenu grâce à l'essor de ce secteur. Cette étude diagnostique a permis d'identifier les plantes à effets insecticides utilisées dans les cultures maraîchères au Bénin ainsi que certains contraintes et méthodes de lutte contre les insectes ravageurs. La plupart des plantes citées n'ont pas encore fait l'objet d'études poussées. Des travaux futurs devront isoler les substances actives de chaque espèce et identifier les ravageurs de spéculation ciblés.

\section{Remerciements}

Nos remerciements vont à l'endroit de l'Agence Universitaire de la Francophonie (AUF) pour son appui finan-cier (Projet de Recherche Partenarial 2018).

\section{References:}

1. Adékambi, S.A., Adégbola, P.Y., Arouna A. (2010). Famers's perception and agricultural technology. The case of botanical extracts and biopesticides in vegetable production in Benin, Third African Association of Agricultural Economists (AAAE) and 48th Agricultural Economists Association of South Africa (AEASA) Conference, Cape Town, South Africa, September 19-23, 2010.

2. Adétonah, S. (2007). Genre, production écologiquement durable et commercialisation des produits maraîchers en zones péri-urbaine et urbaine de l'Afrique de l'Ouest (Bénin, Togo et Ghana). Proposition de recherche. IITA, 16p.

3. Adorgloh-Hessou R. A., 2006. Guide pour le développement de l'entreprise de production et de commercialisation de légumes de qualité dans les régions urbaines et périurbaines du Sud-Bénin. Rapport de consultation IITA-Bénin. 82 p. 
4. Adifon F. H., Azontondé A. H., Houndantode J., Amadji G. L., Boko M., 2015.

5. Evaluation des caractéristiques chimiques des sols sableux du littoral sous-système

6. maraîcher au Sud-Bénin. Annales des sciences agronomiques 19(2) volume spécial : 53-68.

7. Ahouangninou, C., Fayomi, B. E., Martin, T (2011) Evaluation des Risques Sanitaires et Environnementaux des Pratiques Phytosanitaires des Producteurs Maraîchers dans la Commune Rurale de Tori-Bossito (Sud-Bénin). Cahiers Agricultures, 20(3): 216-222. doi:10.1684/agr.2011.0485

8. Akogbéto, M., Djouaka, R., Noukpo, N (2005). Utilisation des insecticides agricoles au Bénin. Bulletin de la société de pathologie exotique, $98: 400-405$

9. Alapini-K., 2009. Evaluation de la pollution du lac Nokoué par les métaux toxiques $(\mathrm{Pb}, \mathrm{Cd}, \mathrm{Hg})$.

10. Mémoire de DESS, CIFRED/ UAC. 77 p.

11. Assogba-komlan, F., Anihouvi, P., Achigan, E., Sikirou, R., Boko, A., Adje, C., Ahle, V., Vodouhe, R., Assa, A. (2007). Pratiques culturales et Teneur en Eléments Antinutritionnels (Nitrates et Pesticides) du Salanum macrocarpon au Sud du Bénin. Africain journal of Food, Agriculture, Nutrition and Developement, 7 , 121

12. Birch, A.N.E., Begg, G.S., Squire, G.R. (2011). How agroecological research helps to address food security issues under new IPM and pesticide reduction policies for

13. global crops production systems. Journal of Experimental botany, Juin, 1-11. doi:10.1093/jxb/err064

14. Broutin, C., Commeat, P.G., Sokona, K. (2005). Le maraîchage face aux contraintes et opportunités de l'expansion urbaine. Le cas de Thies/fandène (Sénégal). Gestion partagée et durable des espaces agricoles et naturels à la périphérie des centres urbains. Document de travail $\mathrm{n}^{\circ} 2$, Ecocité, GRET, 41p

15. CisséI., Fall S. T., Akinbamijo O.O., Diop Y. Mb.,Adediran S.A. 2001.

16. Agriculture urbaine dans les villes ouest-africaines: impacts des systèmes intégrés de production intensive. ISRA/ITC / CRDI.Gardening in the cities of West Africa: Implication for intensive integrated production systems. Workshop/Seminaries atelier.5/8/ Août 2001-09-21

17. Coulibaly, O., Nouhoheflin, T., Aitchédji, C.C., Cherry, A.J., Adegbola, P. (2011). Consumer's perceptions and willingness to 
pay for organically grown vegetables. International Journal of $\begin{array}{lllll}\text { Vegetable } & \text { Science, } & 17 & \text { (4): } & 349-362 \text {, }\end{array}$ doi:10.1080/19315260.2005.563276

18. Diao, M.B. (2004). Situation et contraintes des systèmes urbains et périurbains de production horticole et animale dans la région de Dakar. Cahiers d'études et de recherches francophones/agricultures, 13 (1): 39-49.

19. Doumbia M., Kwadjo K.E., 2009. Pratiques d'utilisation et de gestion des pesticides par les maraîchers en Côte d'Ivoire : Cas de la ville d'Abidjan et deux de ses banlieues (Dabou et Anyama). Journal of Applied Biosciences 18 : 992-1002

20. Guédénon P., 2009. Pollution des écosystèmes par les métaux lourds $(\mathrm{Cd}, \mathrm{Pb}, \mathrm{Cu})$ : Cas du fleuve Ouémé et du lac Nokoué. Mémoire de DESS Sciences de l'environnement et développement durable, CIFRED, UAC, 95p

21. Kanda M. et al., 2009. Le maraichage périurbain à Lomé : pratiques culturales, risques sanitaires et dynamiques spatiales. Cah. Agric., 18(4), 356-363.

22. Kanda M. et al., 2013. Application des pesticides en agriculture maraichère au Togo. VertigO, 13(1), 4-8.

23. Koumolou L., 2009. Bioaccumulation comparée de métaux lourds dans quelques produits maraîchers et champignons comestibles de Cotonou et d'Aplahoué (Bénin). Mémoire de DEA, Environnement, Santé et Développement. 141p.

24. James, B., Atcha-Ahowé, C., Godonou, I., Baimey, H., Goergen, G., Sikirou, R., et Toko, M., 2010. Gestion intégrée des nuisibles en production maraîchère : Guide pour les agents de vulgarisation en Afrique de l'Ouest. Institut international d'agriculture tropicale (IITA), Ibadan, Nigeria. 120 p.

25. Lemay-Boucher, P., Dagnelie, O. (2012). The divorced financial spheres of beninese spouses. Journal of International Development, 1: 1-13, doi:10.1002/jid.2839

26. Madjouma K, Kpérkouma W., Komlan W., Gbandi DB., Adam A., Koffi A., 2009. Le maraîchage périurbain à Lomé : pratiques culturales, risques sanitaires et dynamiques spatiales. CahAgric, vol. $18, \mathrm{n}^{\circ} 4$ :356-363.

27. Moustier, P. (1997), 'Le périurbain en Afrique : une agriculture en marge ?', in Economie Rurale, $\mathrm{n}^{\circ} 241$, 48-55Moustier P., Mbaye A., 1999. Introduction générale. In : Moustier P. et al. (éd.), Agriculture périurbaine en Afrique subsaharienne. Montpellier, France, Cirad, Colloques, 7-17 p 
28. Ogouwalé R., 2007. Système d'irrigation et production maraîchère dans les villes de Cotonou et de Sèmè-Kpdji (Bénin) : Approche cartographique. Laboratoire d'Etudes des climats, des Ressources en eau et de la Dynamique des Ecosystèmes, UAC, Bénin. 21 p.

29. PADAP (2003).Programme d'Appui au Développement Agricole Périurbain au Sud- Bénin, étude de faisabilité. Tome 2, IIED, Bénin 158p.

30. Pazou, E. Y. A., Boko, M., Van Gestel, C. A. M., Ahissou, H., Laleye, P., Akpona, S., Van Hattum, B., Swart, K., Van Straalen, N. M. (2006a). Organochlorine and Organophosphorous 280

31. Pesticide Residues in the Ouémé River Catchment in the Republic of Benin.

32. Environment International, 32(5): 616-623.

33. Pazou, E. Y. A., Laleye, P., Boko, M., Van Gestel, C. A. M., Ahissou, H., Akpona, S., Van Hattum, B., Swart, K. Van Straalen, N. M. (2006b). Contamination of Fish by Organochlo-rine Pesticide Residues in the Ouémé River Catchment in the Republic of Bénin. Environment International, 32: 594-599

34. Temple, L., Moustier, P. (2004). Les fonctions et contraintes de l'agriculture périurbaine de quelques villes africaines (Yaoundé, Cotonou, Dakar). Cahiers Agricultures, 18(1): 15-22.

35. Tokannou, R., Quenum, R. (2007). Etude sur le sous-secteur maraîchage au sud Bénin, Rapport final, AD consult, PAIMAF, $122 \mathrm{p}$.

36. Ton, P., Tovigan, S., Vodouhe, S.D. (2000). Intoxications et Morts au Bénin par l'Endosulfan. In: Pesticides \& Alternatives. Bulletin de Pesticide Action Network Africa, N. 10-Avril, 2000. 